\title{
A Study on the Application of Mobile Medical APP in Medical Data Mining
}

\author{
Xuan Zheng ${ }^{1, a}$, Xuejiao Chen ${ }^{2, b}$ \\ ${ }^{1}$ College of Information Engineering, Gannan Medical University, Ganzhou City, Jiangxi Province, \\ 341000, China \\ ${ }^{2}$ Library of Gannan Medical University, Ganzhou City, Jiangxi Province, 341000, China \\ aemail: \\ bemail:
}

Keywords: mobile medical App; patient health; big data; data mining

\begin{abstract}
With the development of information technology in medical field, a large amount of medical health data has been generated, including personal health records, electronic medical records and other personal health information. These data can provide personalized medical health management programs for the statistics and analysis of medical health data, therefore, the demand for analysis and application of medical and health big data is more urgent. Based on the author's learning and practical experience, this paper first analyzed the concept and characteristics of mobile medical care, and then studied the process of medical data mining, and finally designed a medical data mining system. The results of this study can reshape the medical service process and improve the quality of medical service to a certain extent.
\end{abstract}

\section{Introduction}

With the popularity of mobile Internet and smart phones, mobile medical service is rapidly entering and affecting people's daily life. Mobile health care will be a hot spot in the coming years and will be a new type of medical service,which can change the traditional way of medical treatment. Mobile medical App (Mobile Medical Application) is third party applications based on Mobile Smart phones, and it has been used for medical services and is a form of mobile medical implementation. Mobile medical applications in patient health data mining are convenient for patients to understand their health level, at the same time, it has opened new directions for mobile medical App applications.

\section{Related Concepts and Characteristics of Mobile medical}

Taking patients as the main body. By docking with the hospital information system data, mobile App takes patient ID number as the only master index, and all previous inspection, laboratory, pathology, physiology, medical records and other health data will be taken data mining analysis to obtain more complete health status information and disease early warning information of the same patient, and to generate an online patient health assessment report.

With mobile App platform, the health level assessment content is immediately online pushed according to the patient's needs, so that patients can get an authoritative and professional health report about themselves at any time. Using the mobile App platform, health assessment content will be pushed immediately online, according to the needs of patients, so that patients can always get an authoritative and professional health report on their own. At the same time, pushing professional health knowledge about related diseases via mobile App platforms can let patients fully understand the cause of disease, disease development, drug specifications, rehabilitation process. Taking the 
patient as the subject is to take the patient as the center to organize and design the health information data, to solve the issues concerning to most patients. Mobile medical App has important practical significance in health assessment report and disease information push for patients understanding and early rehabilitation.

Taking doctors as the main body. Docking with the data of hospital information system, mobile medical App to analyze the medication, examination, surgery, and treatment of a large number of patients, it can be used to treat the same disease with different drugs, to study whether the same disease performs same under the same means of examination, and to check the different treatments for the same disease cure effect of information, finally it can summarize the clinical classic treatment experience. For young doctors, these experiences can help them to better understand the book knowledge and clinical practice knowledge, and to grow rapidly. At the same time, we can also find the existing problems from the big data analysis, to make the clinical experience more perfect, and improve the doctor's level of diagnosis and treatment. Mobile App mining individual patient gene profiles and complete medical history data allows for more accurate tracking of disease progression. In the diagnosis and treatment of patients, it can provide more accurate auxiliary diagnosis and treatment information for the doctor in charge, which is helpful for accurate treatment of patients. Taking the doctor as the main body is to regard the doctor as the center to organize and design data, making doctors easily access clinical frontline practice knowledge in routine work and learning processes. This knowledge can be used as a clinical knowledge base to assist in the diagnosis and treatment of the patients, which is of positive significance to the scientific research and teaching of doctors.

\section{The Medical Data Mining Process}

The definition of demand. Requirements analysis and resource discovery are performed at this stage to identify medical goals and key populations, and understand the current solutions to this need. The existing resources are evaluated, then the mining target is determined, the mining plan is made, a subset of data is extracted from the original data, and a data mining database is built.

Data preprocessing. Determine the data source for mining, including data sources in the database or the database external data collected separately. Lists data types, including data attributes, sizes, and formats to describe data sources, to make sure the initial data can partly answer the purpose of data mining, to be also possible to affirm the initial hypothesis or to produce a search for new features. Then selecting the data source in two ways: One is the sampling of the data space, the data selection is random; the other is a sampling of the feature space, the data with some features can be selected.

Data mining. Using appropriate data mining algorithms to perform data analysis is a critical step in the process of medical knowledge discovery. Data mining methods include concept description, association analysis, classification and prediction, clustering analysis, trend analysis, outlier analysis and deviation analysis, etc.. The mining results in the practical application need repeated confirmation, to test its rationality, to establish extensive exchanges between needs and experts, and all the conclusions and their medical experience should verify the rationality to test mining method.

Analysis and evaluation. Assess the accuracy of the knowledge as an example, and compare with the original research goal, the conclusion must be understandable to the user. In the medical field, it is necessary to assess whether the knowledge excavated is new and valuable at the same time. It should be used for medical interpretation of the conclusions and meet the initial goal of data mining 
Identify implementation plans. After the model is built and validated, two methods are used to implement the model: One is to provide the analyst with reference, and then to follow the implementation plan by observing and analyzing the model; Another is to apply the model to different data sets.

\section{Design of Medical Data Mining System}

The system consists of four parts: user interface, database interface, decision tree generation and decision module. The whole structure is shown in Figure 1

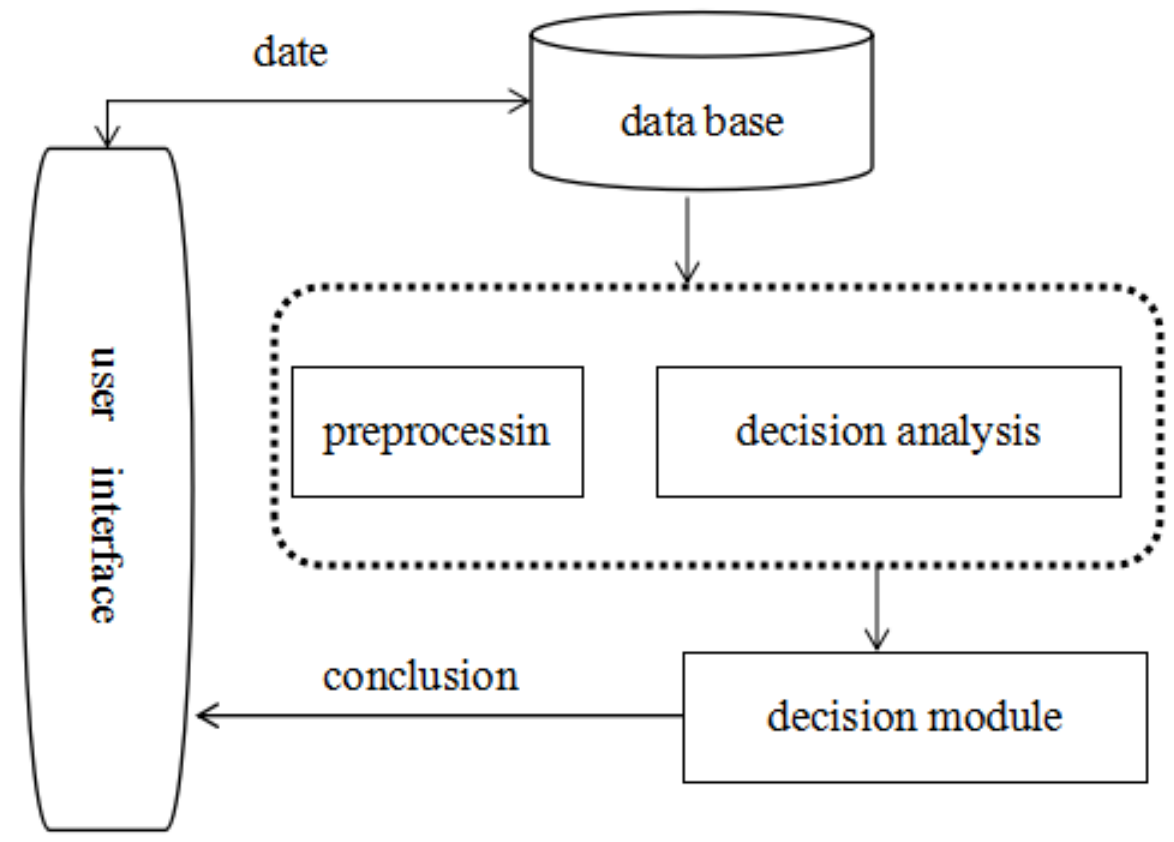

Fig.1 The structure of medical data mining system

Key technology.Data preprocessing and fusion: Medical databases contain vast amounts of different sources of raw information, including a large number of vague, incomplete, noisy, redundant information. Prior to data mining, the information must be cleaned up and filtered to ensure consistency and certainty of data and to translate it into a form suitable for mining. Medical data contains a large amount of text, symbols, digital information, the different physical properties of medical data should be treated by different techniques, so that the convergence can be in property or consistency. Because of the coexistence of multi-source, sequential and non sequential data, the coexistence of digital and non digital data increases the difficulty of information fusion.

Decision tree algorithm: The decision tree algorithm is used to mine medical data. The decision tree algorithm constructs a tree based on the concept of classification. The tree predicts the column values based on the remaining columns of the training set, so each node in the tree represents a particular instance of a column. This node made by algorithm where the decision, and may represent different instances of each column nodes at different depth. The decision tree is to display the classification tree structure, tree structure in which the nodes represent further for data classification.

Data mining analysis example. Mining analysis is divided into three stages: the first stage is data preparation, that is, to collect information about the patient's medical expenses to form a basic database; The second stage is the pretreatment stage, which deals with the basic information and expense information of patients, to form association rules and tables; In the third stage, the decision tree algorithm is used for data mining of medical expense knowledge discovery. The cost data of 
1525 patients in the hospital were analyzed in this case, the medical expenses of patients under 20-70 years of age increase by an increasing rate according to the age group. Among them, 40 to 70 years of age patients accounted for $58.61 \%$ of the total cost of patients. If coupled with gender, the effect of age and gender on hospital costs can be expressed as a decision tree shown in Figure 2.

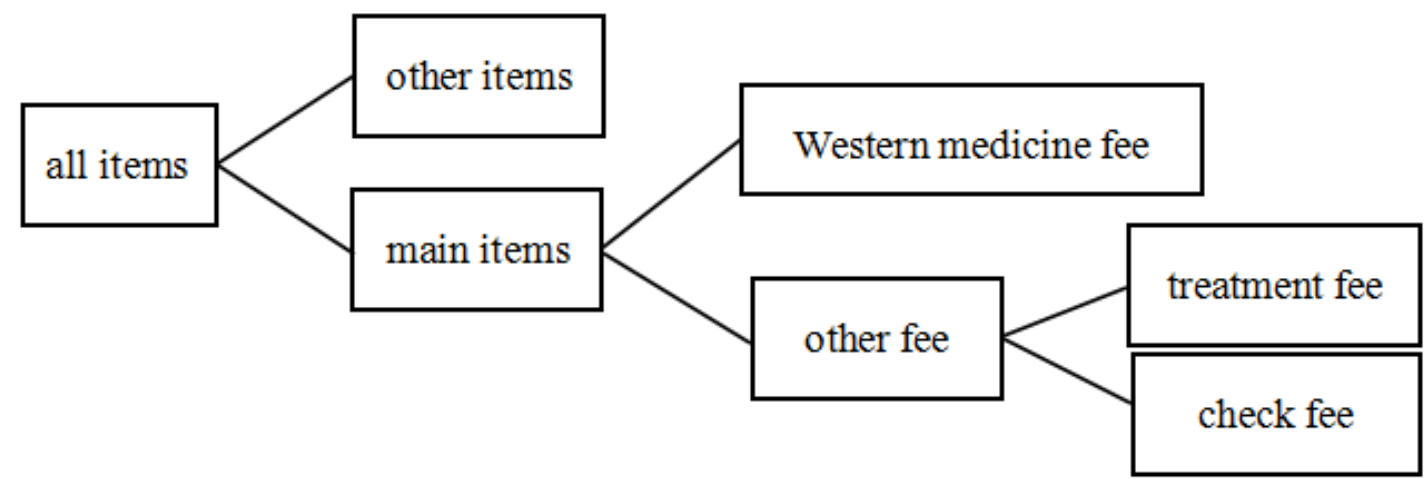

Fig.2 hospital cost decision tree

Where the border represents the density of events, the thicker the border, the more events it represents. For these patients hospitalization expenses of a project schedule, medicine fees, treatment fees, inspection fees accounted for $76.71 \%$ of the total expenses, which are the main component of the most influential factor in the cost of hospitalization, as shown in Figure 3, it is a project cost of hospitalization decision tree, and decision makers can strengthen the medicine fee, treatment fee control, inspection fees to control medical expenses.

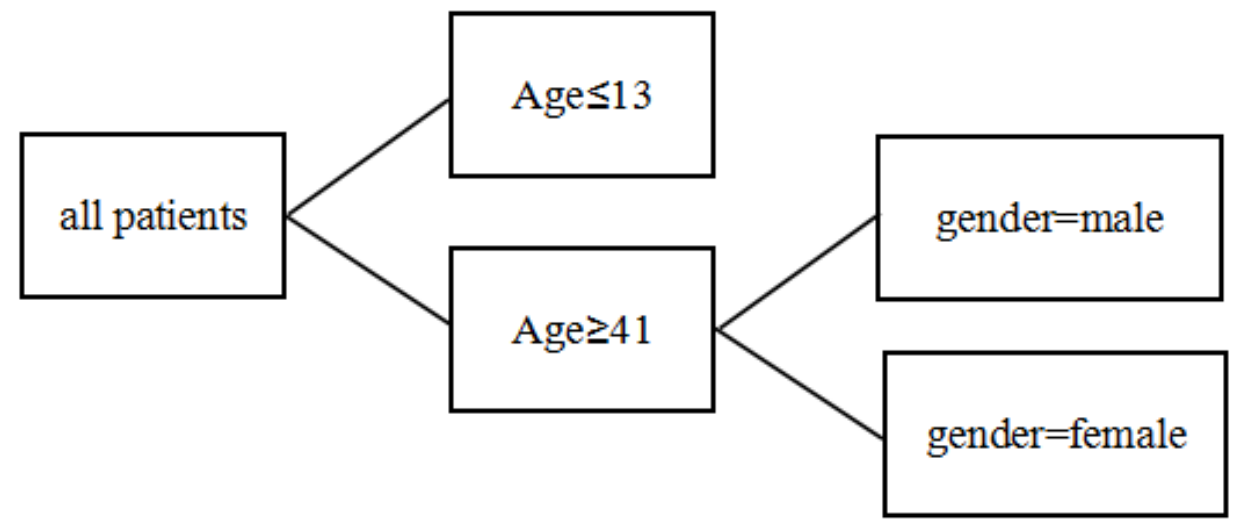

Fig.3 The project cost of hospitalization decision tree,

\section{Summary}

The application of mobile medical App brings new feelings to patients in medical treatment and health care, and it will meet the new needs of health information and health awareness. The solution presented in this paper combined patient care with hospital health services and hospital treatment data. Through the excavation and analysis of patients' large amount of data, it provides deeper data information application services for patients, doctors, hospitals and other groups. It is of great significance to construct advanced medical diagnosis and decision support system by using data mining technology to improve the medical management level of hospital. 


\section{Acknowledgement}

Youth Study Project of University Humanities and Social Sciences in Jiangxi Province: A Study on the Mobile Medical Health Services Applications on Medical Large Data Processing(JC162005).

\section{References}

[1] Yan Xu. Application of machine learning technology in medical data mining[J]. Information Systems Engineering, 2016, (01): 89.

[2] Chen Yi. Application of machine learning technology in medical data mining[J]. Electronic Test, 2015, (03): 93-95.

[3] Yan Yan, Qin Xingbin. Medical health large data research summary[J]. Scientific Research Information Technology and Application, 2014, (06): 3-16.

[4] Liu Chanzhen, Wang Youjun. Research on medical data mining technology and application[J]. Journal of Biomedical Engineering, 2014, (05): 1182-1186.

[5] Bi Jianxin. Application of data mining technology in group medical insurance[J]. Journal of Zhejiang Wanli University, 2006, (05): 22-25.

[6] Yang Haiqing. Application of data mining technology in hospital management[J]. Chinese Journal of Hospital Management, 2005, (07): 497-499. 